\title{
Crystal Structure and Magnetic Properties of Post-Annealed Ba-Ferrite Thin Films for High Density Recording Media
}

\author{
A. Morisako, X. Liu, F. Wada, S. Takei, M. Matsumoto and M. Naoe* \\ Department of Information Engineering, Shinshu University, 500 Wakasato, Nagano 380, Japan \\ * Department of Physical Electronics, Faculty of Engineering, Tokyo Institute of Technology, \\ 2-12-1 O-okayama, Meguro-ku, Tokyo 152, Japan
}

\begin{abstract}
Hexagonal Ba-ferrite films have been prepared at room temperature by facing targets sputtering(FTS) system and they were annealed to crystallize at $900^{\circ} \mathrm{C}$ in air. In this paper, the dependence of the annealing time on grain size of post-annealed Ba-ferrite films has been studied. It was found that the annealing time of $45 s e c$ is sufficient for the crystallization without apparent grain growth under the temperature of $900^{\circ} \mathrm{C}$ and the post-annealed Ba-ferrite films exhibit isotropic magnetic property.
\end{abstract}

\section{INTRODUCTION}

Hexagonal Ba-ferrite(BaM) thin films have many excellent properties such as high coercivity, chemical stability, and mechanical durability to be an ultra high density recording media. In perpendicular recording mode, $c$-axis of hexagonal crystallite should be oriented perpendicularly to the film plane with small c-axis dispersion angle. In order to prepare $\mathrm{BaM}$ films with c-axis orientation, the substrate temperature should be elevated up to $620^{\circ} \mathrm{C}[1]$. Such a high temperature deposition is not applicable for mass production of the recording media. Recently, a few studies on the post-annealing effects for amorphous BaM films prepared by sputtering have been reported to develop a new fabrication technique[2-3].

Our present work is focused on polycrystalline Ba-ferrite films with small grain size and high coercivity as well as high squareness ratio. In this study, the films were prepared on $\mathrm{SiO}_{2} / \mathrm{Si}$ wafer by using the FTS system at room temperature and they were annealed at $900^{\circ}$ in air and the effects of the annealing time on the crystallographic characteristics and magnetic properties of the post-annealed Ba-ferrite films were studied.

\section{EXPERIMENTAL PROCEDURES}

The films have been prepared by FTS system at room temperature with the thickness ranging from 1500 to 1800 Aand they were successively annealed at $900^{\circ} \mathrm{C}$ in air. The substrate is thermally oxidized silicon wafer and the targets were sintered ferrite disk with stoichiometric composition of BaM. The sputtering gas pressure was $2 \mathrm{~m}$ Torr and partial oxygen gas pressure was $0.2 \mathrm{~m}$ Torr. The applied power for the deposition of BaM films was $40 \mathrm{~W}$ and the deposition rate was $8 \AA / \mathrm{min}$. The films were characterized by XRD, VSM, SEM and AFM.

\section{RESULTS AND DISCUSSION}

The as-deposited films were non-magnetic and amorphous. No diffraction lines were observed in the films after annealing for $40 \mathrm{sec}$ at $900^{\circ} \mathrm{C}$. The diffraction lines from hexagonal BaM crystallite were observed in the films after annealing for longer than $45 \mathrm{sec}$. When the annealing time was increased, the relative intensity of the diffraction line from c-plane was increased and a preferential orientation of c-axis was observed in the post-annealed BaM films[3]. This c-axis orientation is not perfect because there are diffraction lines from other plane and the c-axis dispersion angle $\left(\Delta \theta_{50}\right)$ is larger than $20^{\circ}$. This means that the easy axis of magnetization of the post-annealed BaM films has wide distribution in the normal to film plane.

Figure 1 shows the dependence of $\mathrm{M}_{s}$ on the annealing time. The $\mathrm{M}_{s}$ of the films annealed for 30 sec was almost zero and increased from 150 to $260 \mathrm{emu} / \mathrm{cc}$ with the increase of annealing time from 45 to $1200 \mathrm{sec}$. 


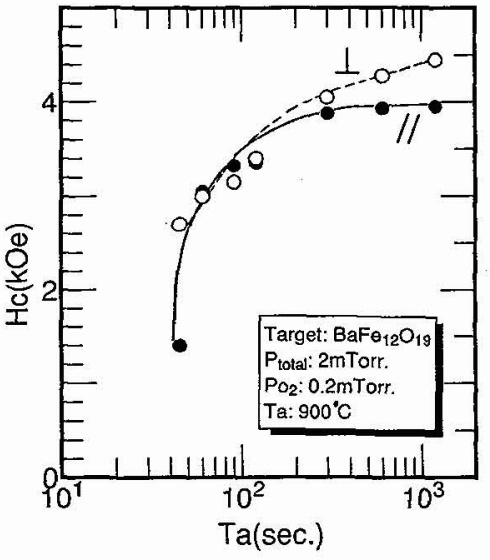

Figure 1: The dependence of $\mathrm{M}_{s}$ on annealing time.

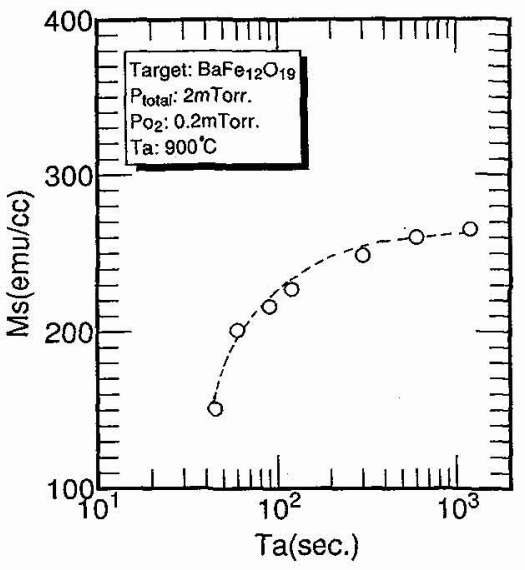

Figure 2: The dependence of coercivity on annealing time.

Figure 2 shows the dependence of coercivities in both in-plane $(\|)$ and perpendicular $(\perp)$ directions. The $H_{c} \|$ increases rapidly from 1.4 to $3.2 \mathrm{kOe}$ and $\mathrm{H}_{\mathrm{c}} \perp$ increases 2.7 to $3.3 \mathrm{kOe}$ with increase of annealing time from 45 to $120 \mathrm{sec}$ and they increase gradually with further increase of annealing time longer than 300 sec. As shown in this figure, the values of $\mathrm{H}_{c} \|$ is almost same as or slightly lower than that of $\mathrm{H}_{c} \downarrow$. This is due to the wide distribution of the easy axis of magnetization(i.e. c-axis of hexagonal crystallite) in normal to film plane, as mentioned above. From these results it was found that the post-annealed BaM films can be applicable for high density longitudinal recording media.

Figure 3 shows the surface morphology of films for different annealing time $\left(t_{a}\right)$. The as-deposited film exhibits extremely smooth surface, as shown in Fig.3(a) and a fine grains was created after the crystallization, as shown in Fig.3(b). When the annealing time is longer than 90sec, apparent grain growth can be observed in Fig.3(c) and (d). Similar results have also obtained by AFM observation. For ultra high density magnetic recording media, the grain size should be small to reduce the noise in reproduced output. From these results, it was found that the annealing time of about $120 \mathrm{sec}$ at the temperature of $900^{\circ} \mathrm{C}$ is enough to crystallize without the grain growth.

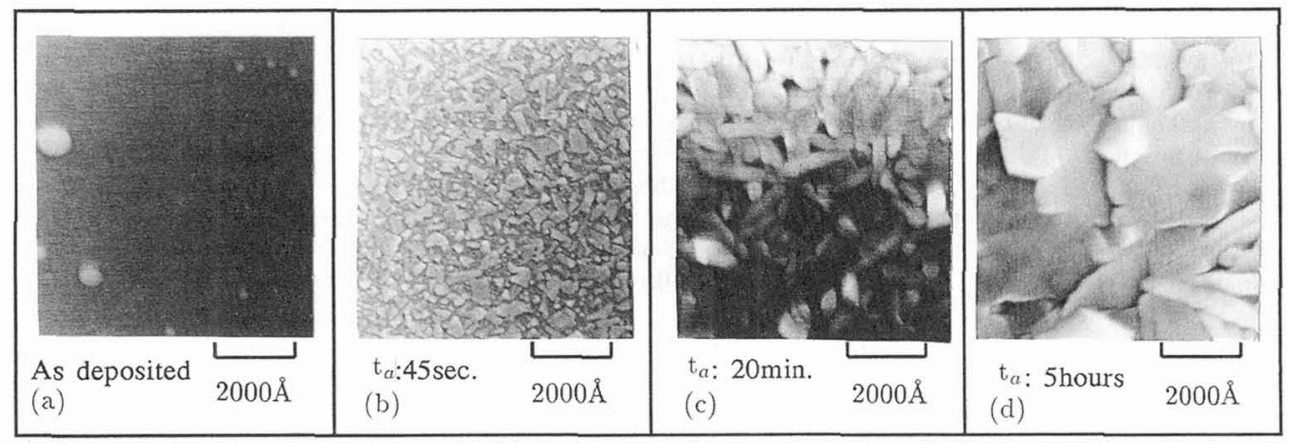

Figure 3: The surface morphology of films for different annealing time by SEM.

\section{CONCLUSION}

In this study, the effects of annealing time at the annealing temperature of $900^{\circ} \mathrm{C}$ on the crystallographic characteristics, the magnetic properties and the surface morphology of the post-annealed BaM films have been studied. The films were crystallized by annealing for about $45 \mathrm{sec}$ and further increase of annealing time results in the apparent grain growtl.

The post-annealed BaM films, which were prepared by rapid annealing, exhibit isotropic magnetic properties and small grain. These films can be applicable for high density recording media.

\section{References}

[1] A.Morisako, M.Matsumoto and M.Naoe: IEEE Trans. Magn., MAG-23, No.5,(1987)2359-2361.

[2] T.L.Hylton, M.A.Parker, K.R.Koffey, R.Umphress, and J.K.Howard, J. Appl. Phys.,vol.75, (1994) 5960-5965.

[3] A.Morisako,M.Matsumoto and M.Naoe:J.Appl.Phys., Vol.79, No.8,(1996) 4480-4482. 